\section{Biogenic nanoparticles: production, characterization, and application of bacterial magnetosomes}

To cite this article: Claus Lang and Dirk Schüler 2006 J. Phys.: Condens. Matter 18 S2815

View the article online for updates and enhancements.

\section{Related content}

\author{
Magnetosome chain superstructure in \\ uncultured magnetotactic bacteria \\ Leida G Abraçado, Fernanda Abreu, \\ Carolina N Keim et al. \\ Energy losses in mechanically modified \\ bacterial magnetosomes \\ Matus Molcan, Hubert Gojzewski, Andrzej \\ Skumiel et al. \\ - Thermostable trypsin conjugates \\ immobilized to biogenic magnetite show a \\ high operational stability and remarkable \\ reusability for protein digestion \\ M Peová, M Šebela, Z Marková et al.
}

\section{Recent citations
The metal binding site composition of the cation diffusion facilitator protein MamM cytoplasmic domain impacts its metal responsivity
Shiran Barber-Zucker et al
Bacterial synthesized metal and metal salt nanoparticles in biomedical applications: An up and coming approach
Yugal Kishore Mohanta et al
Magnetic Nanoparticles as MRI Contrast Agents
Ashish Avasthi et al




\title{
Biogenic nanoparticles: production, characterization, and application of bacterial magnetosomes
}

\author{
Claus Lang and Dirk Schüler ${ }^{1}$ \\ Max Planck Institute for Marine Microbiology, Celsiusstrasse 1, 28359 Bremen, Germany \\ E-mail: dschuele@mpi-bremen.de
}

Received 26 May 2006, in final form 17 July 2006

Published 8 September 2006

Online at stacks.iop.org/JPhysCM/18/S2815

\begin{abstract}
The ability of magnetotactic bacteria (MTB) to navigate along magnetic field lines is based on unique nanosized organelles (magnetosomes), which are membrane-enclosed intracellular crystals of a magnetic iron mineral that assemble into highly ordered chain-like structures. The biomineralization of magnetosomes is a process with genetic control over the accumulation of iron, the deposition of the magnetic crystal within a specific compartment, as well as the assembly, alignment and intracellular organization of particle chains. Magnetite crystals produced by MTB have uniform species-specific morphologies and sizes, which are mostly unknown from inorganic systems. The unusual characteristics of magnetosome particles have attracted a great interdisciplinary interest and inspired numerous ideas for their biotechnological application. In this article, we summarize the current knowledge of magnetosome biomineralization in bacteria. In addition, we will present results on the mass production, as well as the biochemical and physico-chemical analysis and functionalization of bacterial magnetosomes, with emphasis on their characterization as a novel class of magnetic nanoparticles. Finally, we describe the potential of magnetosomes in various biomedical and technological applications.
\end{abstract}

(Some figures in this article are in colour only in the electronic version)

\section{Introduction}

An intriguing example for the biological synthesis of nanoparticles is the biomineralization of magnetosomes. These structures are formed intracellularly in magnetotactic bacteria (MTB) (figure 1(a)), in which they serve as a navigational device for spatial orientation in marine and freshwater habitats by interaction with the earth's magnetic field [1]. Only recently, techniques

1 Author to whom any correspondence should be addressed. 


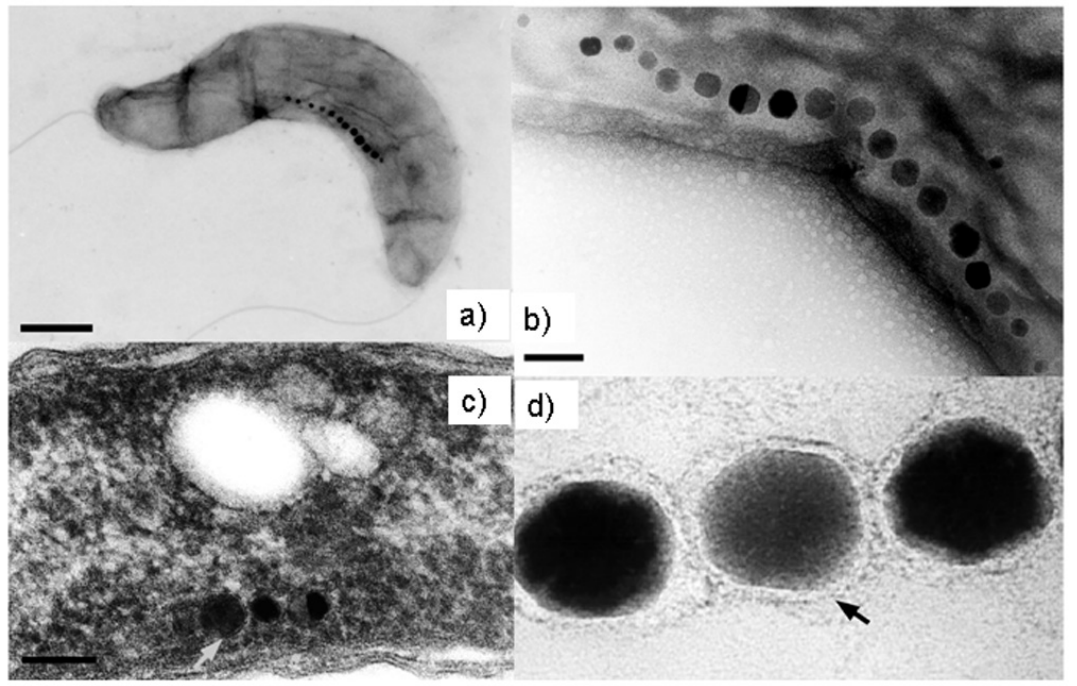

Figure 1. Transmission electron micrographs of negatively stained cells of $M$. gryphiswaldense displaying the magnetosome chain and isolated magnetosomes. (a) Single M. gryphiswaldense cell with the magnetosome chain localized at mid-cell, (b) enlarged view of the magnetosome chain, (c) ultrathin section of a $M$. gryphiswaldense cell with magnetosomes, (d) isolated magnetosome particles with intact magnetosome membranes. The magnetosome membrane is indicated by arrows. The bars are $0.5 \mu \mathrm{m}$ (a) and $0.1 \mu \mathrm{m}$ ((b) and (c)).

have become available for the production and isolation of magnetosomes. The particles represent a new class of magnetic nanoparticles with exceptional properties. Magnetosomes provide numerous attractive possibilities in various applications, due to their unique magnetic and biochemical characteristics.

\subsection{Biomineralization and structure of magnetosomes}

Magnetosomes are membrane-enclosed inorganic crystals consisting either of the magnetic minerals magnetite $\left(\mathrm{Fe}_{3} \mathrm{O}_{4}\right)$ or greigite $\left(\mathrm{Fe}_{3} \mathrm{~S}_{4}\right)$ [1-3]. The particles are usually arranged along the cell axis in one or multiple chains (figure 1) that are oriented with the [lll 111$]$ magnetic easy axis along the chain direction [4], and are organized along a cytoskeletal filamentous structure [5, 6]. Particle sizes are typically $35-120 \mathrm{~nm}$, which is within the singlemagnetic-domain size for magnetite and greigite [7, 8]. A large variety of crystal morphologies such as cubo-octahedral, elongated hexagonal prismatic, and bullet-shaped morphologies were reported [9]. It was shown by high resolution transmission electron microscopy, electron diffraction and electron holography that the morphologies of magnetite crystals in magnetosomes are derived from combinations of the isometric forms $\left\{\begin{array}{lll}1 & 1 & 1\end{array}\right\},\left\{\begin{array}{lll}1 & 1 & 0\end{array}\right\}$ and $\left\{\begin{array}{lll}1 & 0\end{array}\right.$ $0\}$. Morphological variations such as elongated and prismatic structures are due to anisotropic crystal growth [10]. The morphology, size and intracellular organization of magnetosome crystals is subject to a species-specific biological control, which is genetically regulated by a complex and specific set of genes that have been identified within the 'magnetosome island' within the genome of MTB [11, 12]. For magnetite biomineralization, iron is taken up as $\mathrm{Fe}(\mathrm{III})$ or $\mathrm{Fe}$ (II) from the medium and subsequently transported to the magnetosome vesicle [1]. Presumably, a part of the iron is then reoxidized to form a highly reactive Fe(III) oxide, probably ferrihydrite, which may react with dissolved Fe(II) to form magnetite by a via- 
solution process [13]. Compartmentalization through the formation of membrane vesicles enables the processes of magnetite mineralization to be regulated by biochemical pathways. The magnetosome membrane (MM) is crucial in the control of crystal growth by providing spatial constraints for shaping of species-specific crystal morphologies. Biomineralization of magnetite requires a precise regulation of the redox potential, $\mathrm{pH}$, and the prevalence of a supersaturating iron concentration within the vesicle. Thus, the MM performs specific functions in the transport and accumulation of iron, nucleation of crystallization, and redox and $\mathrm{pH}$ control $[14,15]$.

\subsection{Applications of magnetosomes}

Bacterial magnetic nanoparticles have been suggested for a number of in vitro applications, such as magnetic separation and procedures for labelling and immobilization of various biomolecules. The use of magnetosomes has been described for numerous purification procedures such as the extraction of mRNA and DNA from biological samples such as tissues, blood and bacterial cells. For instance, the efficiency of DNA recovery with dendrimer-modified magnetosome particles was sixfold higher with bacterial particles than with artificial magnetic particles [16]. The automation of a DNA extraction procedure based on dendrimer-modified particles has been reported recently $[16,17]$. The isolation of mRNA was facilitated by oligo(dT)-modified magnetosomes [18]. Magnetosomes similarly modified with oligonucleotides have been employed in an automated magnetic microarray for the detection of different cyanobacterial DNA with genus-specific probes [19].

Another set of biotechnological applications is based on the immobilization of proteins, peptides and enzymes on magnetic particles, which allows the selective separation and reuse of immobilized enzymes from a reaction mixture. Compared to micrometric particles, the use of nanosized particles is preferred due to (i) their higher specific surface area and therefore higher binding capacity and (ii) their lower mass transfer resistance. Because of their large surface-tovolume ratio, bacterial magnetosome particles were successfully harnessed for immobilization of the enzymes glucose oxidase and uricase in early experiments [20]. Likewise, the immobilization of immunoglobulins has received great attention and inspired the development of diverse applications. One approach of immunoglobulin immobilization on magnetic particles relied on chemical cross-linking of the antibody with the MM [21]. Another approach attempted the genetic modification of magnetosome membrane proteins (MMP) to generate protein fusions of a MMP and an immunoglobulin binding protein such as the staphylococcal protein A or streptococcal protein $\mathrm{G}[22,23]$. The second approach is potentially superior to chemical cross-linking, as the antibody is oriented more accurately, although it remains to be shown that the polypeptides used for the genetic fusion are in fact native constituents of the MM. Antibody-magnetosome conjugates were employed for automated immunoassays to detect environmental pollutants, hormones and toxic substances [22, 24]. In addition, antibodymodified magnetosomes have been used successfully for the specific separation of target cells from human blood [25]. Another application is the use of streptavidin-modified magnetosomes for the automated discrimination of single nucleotide polymorphism. The streptavidin-modified particles were coupled to biotinylated oligonucleotides to facilitate magnetic separation of DNA hybrids. Single nucleotide polymorphisms were detected as decreased fluorescent intensities in a fluorescence resonance energy transfer (FRET) system with FITC (donor)-labelled DNA and a POPO-3 (acceptor), which preferentially intercalates double stranded sequence of complementary strands [26]. The magnetic properties of the particles can not only be used for the purification and immobilization of biomolecules but also for their detection. Magnetic force microscopy was used for the highly sensitive detection and quantification of streptavidin 
immobilized on glass slides with biotin conjugated magnetosomes [27]. The magnetosome chain is one of the most complex and highly ordered structures found in a bacterium, and it has been suggested that biomimetic approaches could be used for the fabrication of self-assembling magnetic nanostructures inspired by magnetosome chains, such as magnetic nanowires and nanotubes as building blocks in magnetic devices. In fact, it has been recently shown by Banerjee and co-workers that magnetic nanotubes can be assembled by the incorporation of isolated bacterial magnetosomes into peptide nanotubes [28].

In summary, these examples impressively demonstrate the tremendous biotechnological and nanotechnological potential of bacterial magnetic nanoparticles. However, numerous fundamental questions, that have remained unsolved thus far, have prevented an application of magnetosomes at technical scale. Thus, our project aimed to produce and thoroughly characterize bacterial magnetosome particles for their use in a number of applications. This was done in a combined and collaborative approach, which is described in the following.

\section{Production of magnetosome particles}

Previous attempts to characterize and apply magnetosome particles were hampered by their limited availability. In addition, the particles were poorly characterized in terms of their biochemical and physico-chemical properties. The efforts of our group to establish protocols for the large scale production of magnetic bacteria and magnetosomes which facilitated the detailed biochemical and physical characterization of magnetosomes are summarized in the following. We also outline novel routes for the generation of functionalized magnetosomes with high technological significance.

\subsection{Development of methods for mass cultivation of MTB}

High yields of magnetosomes from large quantities of cells cultivated under defined growth conditions are required for both the biochemical and biophysical characterization of magnetosomes. MTB are fastidious organisms and the few strains that are available in pure culture are difficult to grow. M. gryphiswaldense (figure 1(a)) was selected as a magnetotactic model organism because it can be grown in simple liquid media containing short organic acids as a carbon source. In addition, this organism is amenable to genetic analysis, and the determination of its genome sequence has been nearly completed [12, 29]. Therefore we sought to establish optimum conditions for the mass cultivation of the microaerophilic $M$. gryphiswaldense in flasks and in a fermenter. The first step was the optimization of the medium to increase cell yield, magnetism, resulting in a defined medium in which high yields of cells and magnetosomes can be obtained at moderate costs.

The magnetotactic strains $M$. magnetotacticum, M. magneticum and $M$. gryphiswaldense produce magnetite only under microaerobic conditions, whereas higher (e.g. atmospheric) oxygen concentrations inhibit growth and repress magnetite formation. Among the strains tested in our study, M. gryphiswaldense exhibited the highest oxygen tolerance, and growth was unaffected by oxygen concentration over a wide range ( 0.25 up to $150 \mathrm{mbar})$. However, we observed an increased mutability within the magnetosome island if cells were grown at higher oxygen levels, leading to the irreversible loss of the capability to form magnetosomes [12]. In initial growth experiments, cells were cultivated in flasks under a microaerobic gas mixture containing $1 \%$ oxygen in $99 \%$ nitrogen [30]. These conditions are only of limited use for growth experiments and large scale cell production, because the oxygen supply cannot be kept constant during the incubation period. In fact, the oxygen partial pressure decreases due to cellular respiration, resulting in a metabolic shift from microaerobic to anaerobic growth 


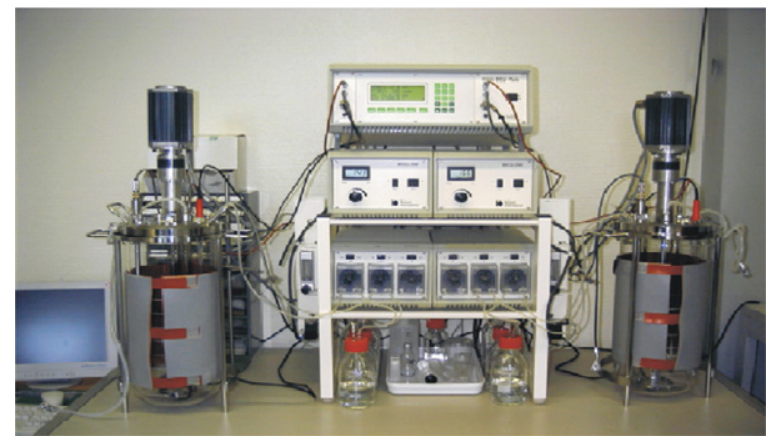

Figure 2. Dual-vessel $(2 \times 10 l)$ laboratory fermenter system for mass culture of $M$. gryphiswaldense

and a decreased growth rate. Hence, the control of a microoxic environment is a crucial parameter for growth and magnetosome formation, which however, cannot be maintained during cultivation in conventional flasks. Therefore, a protocol for mass cultivation of MTB in an automated oxygen-controlled fermenter (figure 2) was established, which allows the continuous maintenance of low oxygen partial pressures [30]. A Biostat A Twin (B. Braun Biotech International, Melsungen Germany) Bioreactor was specifically adapted for the microaerobic cultivation of microaerophilic bacteria under oxystat conditions. This was achieved by the installation of a highly susceptible oxygen amplifier and accessory equipment for the gas supply. Defined low oxygen partial pressures were regulated by a cascade control via separate and independent gassing with nitrogen and air. Nitrogen supply was controlled by means of a flowmeter (DK 46N; Krohne, Duisburg, Germany) installed in line with a pulsed solenoid gas valve (Bürker, Ingelfingen, Germany). The air supply was regulated by a thermal mass-flow controller (BRA-001F; Bronkhorst, Ruurlo, Netherlands), and an additional pulsed solenoid control valve. The switch between nitrogen and oxygen gassing depends on the actual oxygen partial pressure in the medium. At oxygen partial pressures of $0.5 \%$ above the set value nitrogen was sparged into the fermenter at a constant rate and at oxygen partial pressures below $99.5 \%$ of the set value the aeration rate was regulated via the mass-flow controller and the pulsed solenoid valve. The system allows the precise maintenance of $p \mathrm{O}_{2}$ tensions ranging from $0.25-212$ mbar over a wide range of cell densities with fluctuations less then $5 \%$ of the set value. This oxystat fermenter has been used to determine optimal oxygen partial pressures for magnetite production and cultivation of $M$. gryphiswaldense. Magnetite formation occurred only below a threshold value of 10 mbar, whereas it was inhibited at higher oxygen concentrations. We found a clear correlation between the amount of magnetite formed and $\mathrm{pO}_{2}$ exists, and most favourable conditions for magnetite biomineralization at $0.25 \mathrm{mbar}$ (figure 3) [29]. Interestingly, particles grown at 10 mbar displayed smaller sizes (about $20 \mathrm{~nm}$ ) compared to $42 \mathrm{~nm}$ of particles produced under optimal conditions $(0.25 \mathrm{mbar})$, indicating that morphology and size of particles can be controlled by growth conditions. Likewise, reduced particle sizes were obtained under conditions of iron limitation, or if magnetite formation was synchronized by the addition of iron to iron-starved cells shortly before cell harvest.

In a comparative study, a productivity of $6.3 \mathrm{mg}$ magnetite $1^{-1}$ day $^{-1}$ was found for $M$. gryphiswaldense compared to 3.3 and $2.0 \mathrm{mg}$ magnetite $1^{-1}$ day $^{-1}$ for Magnetospirillum sp. AMB-1 and M. magnetotacticum MS-1, respectively [30]. Cultivation of M. gryphiswaldense in the oxystat with the improved medium increased the maximum cell yield to $0.40 \mathrm{~g}$ dry weight $1^{-1}$ compared to $0.33 \mathrm{~g}$ dry weight $1^{-1}$ which were reported earlier [31]. Thus, the protocol 


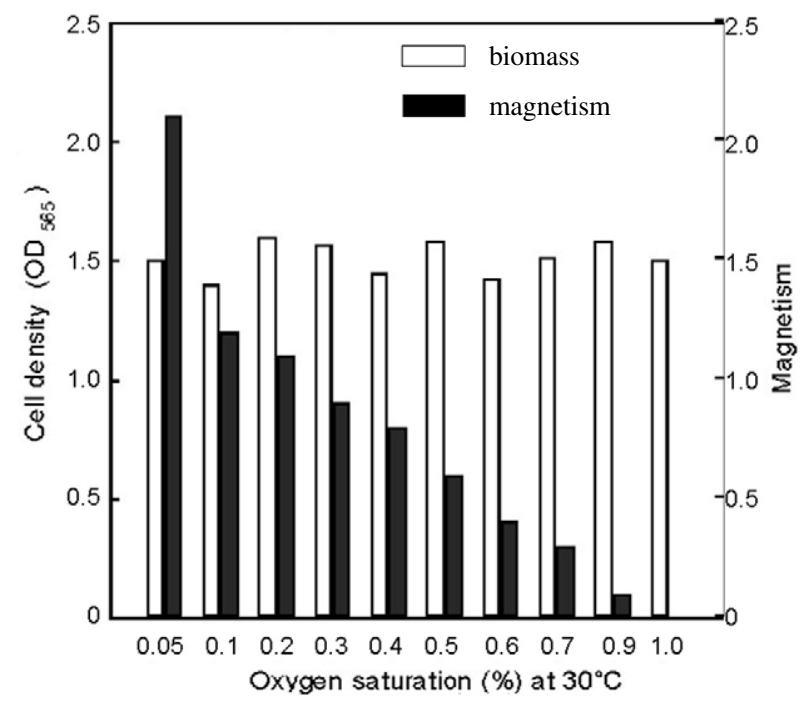

Figure 3. Biomass production (OD) and cell magnetism of oxystat grown $M$. gryphiswaldense at different constant oxygen concentrations.

established for $M$. gryphiswaldense allows mass production of magnetosomes in amounts sufficient for their characterization and applications.

\subsection{Isolation and purification of magnetosomes}

We have developed a protocol for the purification of magnetosomes from M. gryphiswaldense. Magnetosome particles are distinguished from organic cell constituents by their high density and their magnetism. These properties can be harnessed for their purification from cells by a straightforward isolation protocol. After cell disruption by French press and removal of cell debris by centrifugation the magnetosomes can be easily separated from the crude extracts by magnetic separation columns [32]. Magnetic separation is followed by ultracentrifugation into a $55 \%(\mathrm{w} / \mathrm{w})$ sucrose cushion. This procedure results in suspensions of purified magnetosome particles with intact enveloping membrane structures (figure 1(d)). The isolated magnetosomes are relatively stable in the presence of mild detergents. However treatment with strong detergents $\left(1 \%\right.$ SDS at $\left.95^{\circ} \mathrm{C}\right)$ or organic solvents solubilizes the MM, which results in the agglomeration of membrane-free magnetite particles (figure 4) [32, 33].

\section{Characterization of magnetosomes}

\subsection{Biochemical characterization of magnetosomes}

For any functionalization and subsequent application, a detailed knowledge of the biochemical composition and protein content of the isolated magnetosomes is an essential requirement. Therefore, the biochemical characteristics were thoroughly analysed. A number of common fatty acids are present in the MM from M. gryphiswaldense [32, 33]. The most abundant polar lipids in the MM are phosphatidylethanolamine and phosphatidylglycerol which are also the most abundant polar lipids in whole-cell extracts. The fatty acid composition of the $\mathrm{MM}$ is very similar to the fatty acid composition of the whole cell in that the most 


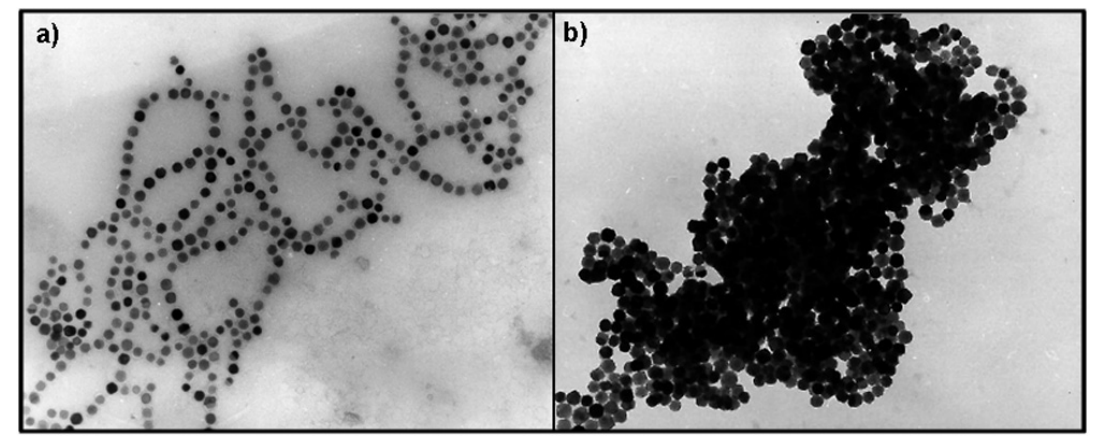

Figure 4. (a) Isolated magnetosome particles with intact MM display a strong tendency for chain formation, whereas removal of the MM by SDS treatment results in the agglomeration of membrane-free particles (b).

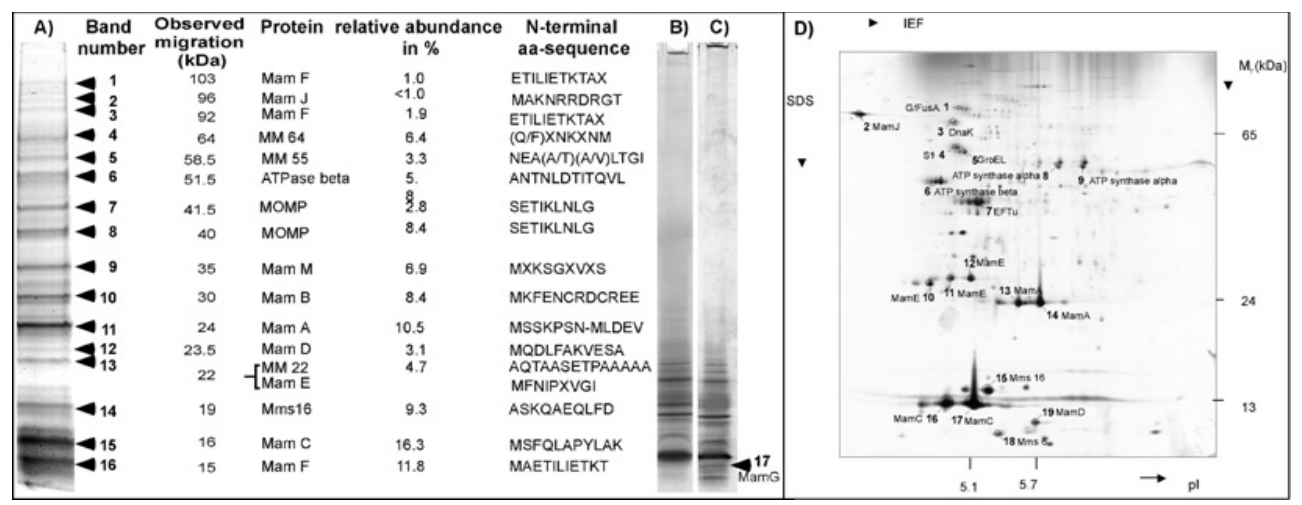

Figure 5. MM-associated proteins separated by one- and two-dimensional PAGE (redrawn after Grünberg et al [32]. (A) Summary of MM proteins detected by Coomassie stain in 1D SDS-PAGE (16\%). Proteins from indicated bands were identified by N-terminal amino acid analysis (Edman degradation). (B) Coomassie and (C) silver-stained SDS-Tricine gels (16.5\%) of MM proteins. (D) Silver-stained 2D PAGE of MM proteins from M. gryphiswaldense. Proteins from marked spots were identified by mass spectrometric sequencing (2D PAGE analysis was performed by R Reszka, MDC Berlin).

abundant fatty acids are identical. However certain fatty acids, that are found in whole cells, e.g. 3-hydroxyhexadecanoic acid (3OH 16:0), 3-hydroxyoctadecanoic acid (3OH 18:0) and 2hydroxydecenoic acid (2OH 18:1), are absent from the MM. These amide-linked fatty acids are typically present in the outer membrane of gram-negative bacteria. These results indicate that the MM originates from the cytoplasmic membrane. This has recently been confirmed directly by cryo-electron tomography, which demonstrated that magnetosomes are membrane invaginations originating from the cytoplasmic membrane [5].

For the identification of magnetosome-associated proteins, the $\mathrm{MM}$ of isolated magnetosomes was analysed as described in detail in [31]. Briefly, the MM was solubilized by boiling in a buffer containing 2\% (w/w) SDS and 5\% (w/v) 2-mercaptoethanol. The samples were subsequently subjected to one-dimensional SDS-polyacrylamide gel electrophoresis (SDS-PAGE) according to the procedure of Lämmli (figure 5(A)) [34] or to Tricine-SDSPAGE according to Schägger and Jagow (figures 5(B) and (C)) [35]. In order to obtain improved protein separation, two-dimensional gel electrophoresis was carried out additionally 
(figure 5(D)) [32]. After electrophoresis the proteins were blotted onto a membrane and the N-termini of separated proteins were sequenced by Edman degradation. Additionally, magnetosome-associated proteins were identified by mass spectroscopy, either after size separation from single spots or bands, or from total tryptic digests of entire magnetosome preparations. For the latter purpose, magnetosomes were reduced with dithiotreitol, alkylated with iodacetamide and digested with trypsin to completion. Afterwards the magnetic moiety was removed, and the supernatant was chromatographically separated using a capillary liquid chromatography system. The eluted peptides were analysed by a Q-TOF hybrid mass spectrometer.

The peptide profiles were correlated with the preliminary M. gryphiswaldense genome sequence [32]. Analysis of the extracted membrane revealed that the magnetosome is associated with a highly specific and complex subset of proteins, which are present in various quantities. The amount of MM-bound polypeptides approximately represents $0.1 \%$ of the total cellular protein [33]. The combination of these proteomic techniques resulted in the identification of 18 major polypeptides in the magnetosome subproteom (figure 5) $[11,12,32,33]$. The different resistance of magnetosome proteins towards proteases and detergents indicates that some proteins are very tightly bound to the magnetosome crystals and/or embedded within the membrane. The highly abundant proteins MamC, MamF and Mms16 which have electrophoretic mobilities corresponding to sizes of 15-19 kDa are the most stable MMP. Others, as for instance MamA, are loosely attached and can be selectively solubilized by mild detergents. Based on sequence analysis, MMP can be assigned to a number of characteristic protein families, which are presumed to perform specific functions in $\mathrm{MM}$ vesicle formation and assembly, iron transport, and control of nucleation and growth of magnetite crystals. Several of the proteins contain covalently bound c-type haeme as revealed by peroxidase staining. No glycoproteins, which are common constituents of other biomineralizing systems, have been detected so far. Both MamB and MamM are members of the CDF (cation diffusion facilitator) family of metal transporters, which comprises proteins that function as efflux pumps of toxic divalent cations, such as zinc, cadmium, cobalt and other heavy metal ions. Specifically, MamB and MamM have greatest similarity to the CDF3 subfamily, which was postulated to comprise putative iron transporters [36]. It has been speculated that MamB and MamM are involved in the magnetosome-directed uptake of iron, and preliminary evidence obtained from mutant analysis seems to support this assumption [52]. MamE and MamO display sequence similarity to HtrA-like serine proteases. The mamP gene, encoding a further deduced protein with similarity to this family, is collocated with mamE and $m a m O$ within the same operon. HtrA-like proteins share a conserved trypsin-like protease domain and one or two PDZ domains. They act as molecular chaperones and heatshock induced proteases, which degrade misfolded proteins in the periplasm [37]. It has been suggested that MamE and MamO are involved in magnetosome formation, perhaps by the processing, maturation and targeting of MMP during MM assembly [33].

The most abundant MM-associated proteins MamC, MamD, MamG, and MamF have no known homologues in organisms other than MTB, and thus represent unique, MTBspecific protein families. One noticeable feature common to several of these proteins is the presence of repetitive motifs. Examples are MamD, Mms6 and MamG, which share conspicuous hydrophobic sequence motifs that are rich in repeated leucine and glycine residues. Similar motifs containing LG-rich repetitive sequences have been found in other proteins that have a tendency for self-aggregration or are involved in the formation of supramolecular structures [38]. The Mms6 protein of Magnetospirillum strain AMB-1 is a tightly bound constituent of the MM, which exhibits iron binding activity and has an effect on the morphology of growing magnetite crystals in vitro [39]. Another conspicuous sequence pattern is found in 
MamJ. This protein displays extensive self-similarity and is particularly rich in repeats of the acidic amino acid residues glutamate and aspartate. These features are typical for other proteins involved in biomineralization processes and originally lead to the speculation that MamJ might mediate nucleation and growth of magnetite crystals. However, the targeted deletion of the mamJ gene revealed its involvement in the alignment of the magnetosome chain in the cell, probably by interaction with a filamentous structure [6].

\subsection{Physico-chemical characterization of magnetosome particles}

The analysis of the physico-chemical and magnetic properties of magnetosomes is of great relevance for technological application, because biogenic magnetosomes can be expected to have unique magnetic characteristics as they have evolved as a navigational device in magnetic bacteria that is sensitive to the geomagnetic field and provides a magnetic moment sufficient to align bacterial cells [40]. The magnetic characteristics of the particles were subject to several studies. The hysteresis of isolated magnetosome particles was measured in different magnetometers by Hergt et al and Eberbeck et al [41, 42]. In the first study a hysteresis corresponding to a coercive field of $6500 \mathrm{~A} \mathrm{~m}^{-1}$ was measured at room temperature. In the study carried out by Hergt et al coercivities of $2600 \mathrm{~A} \mathrm{~m}^{-1}$ were obtained for gelatine immobilized magnetosomes. Hysteresis loops for immobilized magnetosomes further were measured at frequencies between $100 \mathrm{~Hz}$ and $1 \mathrm{MHz}$ in a vibrating sample magnetometer. The coercivity of magnetosomes is extremely high compared to commercially available magnetic nanoparticles (Resovist ${ }^{\circledR}$, trademark of Schering AG, Germany) with $20 \mathrm{~A} \mathrm{~m}^{-1}$ [42].

Another magnetic characteristic of magnetosomes, which is of potential relevance for various applications is their relaxation time. In a fluid state a relaxation time of $80 \mathrm{~ms}$ was measured, which is attributed to Brownian relaxation. Néel relaxation was largely beyond the measurement time window of the instrument which suggests that the particles can be considered blocked [42].

Atomic force microscopy and magnetic force microscopy were used to determine if the blocking behaviour is attributable to the formation of aggregates or if single magnetosomes possess remanent magnetic moments. Particles with a size of about $27 \mathrm{~nm}$ still bear a remanent magnetic moment $[42,43]$. The presence of single magnetosomes was verified with the same methods by Albrecht et al [43].

While mature magnetosome crystals are mostly within the ferrimagnetic size range, the crystal sizes are under biological control and can be genetically modified. For instance, magnetic particles from a mutant exhibit a narrower size distribution and smaller diameters (figure 6) that predominantly fall into the superparamagnetic size range, as revealed by small angle scattering using polarized neutrons (SANSPOL) [44].

So far the analysis of the magnetic properties of the particles has shown that magnetosomes are single-magnetic-domain particles with unique magnetic characteristics, which are hardly matched by synthetic magnetic particles. These properties designate them for medical applications such as hyperthermia and magnetic resonance imaging. An outline of the recent developments in this field will be given in the next section.

\section{Evaluation of magnetosomes for medical applications}

Superparamagnetic nanoparticles are used as magneto-pharmaceuticals for diagnostic purposes. They serve as contrast agents in magnetic resonance imaging to enhance the contrast between normal and diseased tissue or to indicate the status of an organ. Magnetosomebased ferrofluids are promising candidates for magnetic resonance tomography contrast agents, 


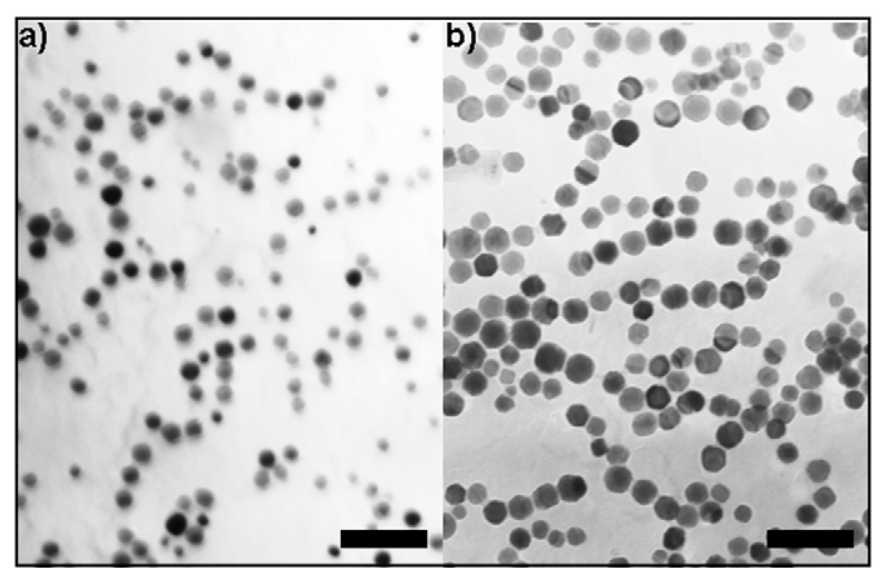

Figure 6. TEM picture of magnetosomes isolated from a $M$. gryphiswaldense mutant strains (MSR1K (a)) with reduced particle size and from the wild-type strain (MSR-1 (b)). The bar size is $150 \mathrm{~nm}$.

which can be detected at very low concentrations in clinically employed MR tomographs. That magnetosomes are an expedient alternative to synthetic ferrofluids was shown in in vitro experiments by Herborn et al [45]. The longitudinal and the transverse relaxivity $\left(R_{1}=7.688 \mathrm{mmol}^{-1} \mathrm{~s}^{-1} ; R_{2}=145.67 \mathrm{mmol}^{-1} \mathrm{~s}^{-1}\right)$ were calculated after studying different magnetosome dilutions in a clinical $1.5 \mathrm{~T} \mathrm{MR}$ tomography $\left(63 \mathrm{MHz}, 21^{\circ} \mathrm{C}\right)$. The low detection limit of magnetosomes in MRI systems can be harnessed for MR molecular imaging. An approach for the twofold labelling of macrophages with fluorescently labelled magnetosomes was presented recently. The modified macrophages may find application in the simultaneous detection of inflammations by near infrared fluorescence microscopy and MRI [46].

Another promising application for magnetosomes might be the method of hyperthermia treatment, in which magnetic nanoparticles are used for controlled tissue heating to promote cell necrosis in tumors. After magnetic nanoparticles are applied to the target tissue, an alternating external magnetic field is applied. Due to loss processes resulting from the reorientation of the magnetic moments of the particles, heat is generated, which results in cell necrosis in tumor cells [47]. The method relies on the development of magnetic nanoparticles with high specific loss powers [41]. In a recent study of magnetosomes from $M$. gryphiswaldense in biomedical applications such as hyperthermia and thermoablation, Hergt and co-workers found exceptionally high specific powers losses $\left(960 \mathrm{~g} \mathrm{~W}^{-1}\right.$ at $10 \mathrm{kA} \mathrm{m}^{-1}$ and $410 \mathrm{kHz}$ ), which substantially exceed the results obtained with artificial particles [41].

\section{Functionalization of magnetosomes}

The encapsulation of the magnetic crystal within the MM provides a natural 'coating', which ensures superior dispersibility of the particles and provides an excellent target for modification and functionalization of the particles. Besides in vivo 'tailoring' of magnetite crystals, also the biochemical composition of the MM can be altered by genetic engineering. A highly promising approach is the design of magnetosomes with functionalized surfaces (figure 7). This can be achieved by the generation of chimeric proteins, which are specifically displayed on the surfaces of isolated magnetosomes. It has been demonstrated that magnetosome proteins can be used for the construction of functional genetic fusions $[48,49]$. In initial experiments we were 


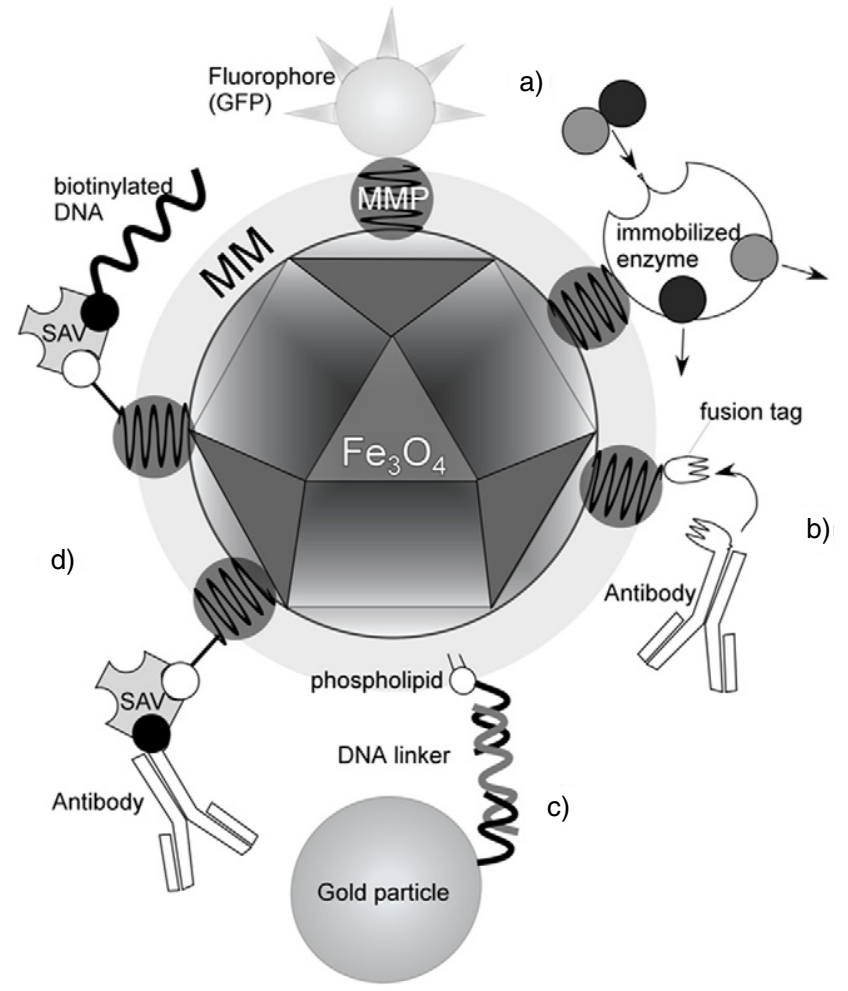

Figure 7. Potential modifications of magnetosome particles by the introduction of different functional moieties resulting in hybrid bacterial magnetic nanoparticles (modified after Lang and Schüler [50]). Magnetosomes could be modified by (a) magnetosome-specific expression of enzyme and fluorophore proteins (e.g. GFP) by genetic fusion to MMP, (b) expression of fusion tags such as intein- or strep-tags as anchor groups for subsequent conjugate formation with various biomolecules, (c) formation of conjugates with gold particles or quantum dots via a DNA linker, (d) biotinylation of membrane lipids and proteins, which would facilitate the subsequent streptavidin-mediated conjugation to various molecules such as nucleic acids or antibodies. MMmagnetosome membrane, MMP_-magnetosome protein, SAV—streptavidin.

able to specifically label magnetosomes with a fusion of a green fluorescent protein and a MMP (figure 8). Replacement of the GFP function with other relevant polypeptide sequences could be used for the introduction of functional moieties, as for instance biomolecular recognition groups such as the biotin-streptavidin system [50].

\section{Conclusions}

Magnetosome formation in MTB provides a novel magnetic nanomaterial that is generated by a mineralization process with control over the chemical composition, morphology, size and intracellular localization of the magnetic mineral. In summary, our work supported by the DFG priority programme 'Colloidal Magnetic Fluids' has contributed to a greatly improved knowledge about the production and characterization of these particles, which will facilitate their future application. In this article, we have highlighted much of the current knowledge about the physiological and genetic basis of magnetosome biomineralization as well as potential uses of magnetosome particles in a number of biotechnological applications. 


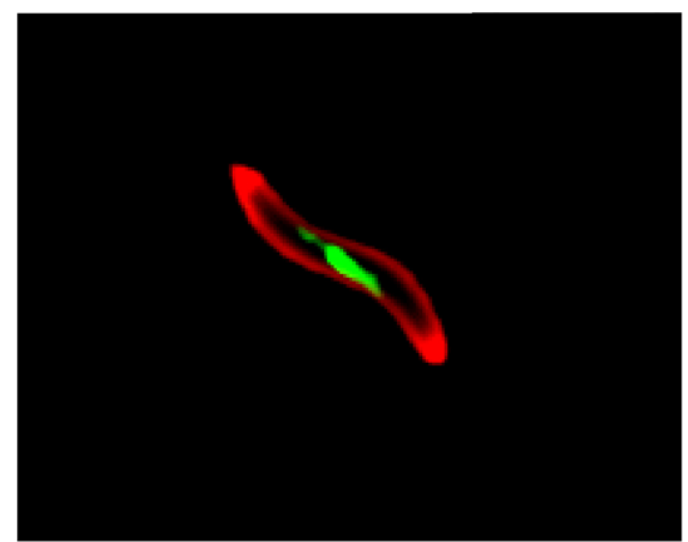

Figure 8. Fluorescent micrograph of $M$. gryphiswaldense expressing enhanced green fluorescent protein (EGFP) fused to a MMP. The green fluorescence signal originates from the magnetosome chain displaying EGFP at mid-cell. Cell membranes (red) were stained with the fluorescent dye FM4-64 (Invitrogen). This demonstrates that heterologous proteins can be fused to functional MMP for magnetosome-specific display. In future this approach could be extended to the immobilization of enzyme proteins or the introduction of protein tags that facilitate secondary in vitro functionalization of magnetosome particles by coupling to a variety of biomolecules.

Beside the establishment of techniques for the improved handling and cultivation of MTB in the laboratory, the establishment of methods for genetic manipulations for several MTB species as well as efforts in the determination of their genome sequence represented major breakthroughs. With the recent advent of genetic technology for transformation and site-directed mutagenesis for MTB [29, 49, 51], genetic analysis has become a powerful tool in the study of magnetosome formation. The capabilities of MTB to precisely control the composition and morphology of inorganic particles have been explored only recently, and have contributed to the development of a new and largely unexplored area based on the use of MTB in biosynthesis of magnetic nanomaterials. The available genetic technology will not only elucidate the pathways of magnetosome formation at molecular level, but also holds great promise for the design of biogenic magnetic nanoparticles with desired properties by genetic engineering. An in vivo 'tailoring' can be applied both to organic and inorganic constituents of magnetosomes. The site-directed mutagenesis of identified iron-transporting magnetosome proteins might be used to generate magnetosome with a modified specificity for the magnetosome-directed uptake of different metals, potentially resulting in inorganic magnetic cores with an altered chemical composition. Likewise, the biochemical composition of the MM may be altered in vivo by genetic engineering. A highly attractive and promising approach will be the design of magnetosomes with functionalized surfaces. This can be achieved for instance by the generation of chimeric proteins, which are specifically displayed on the surfaces of isolated magnetosomes, or by the chemical conjugation of magnetosomes and biomolecules.

\section{Acknowledgments}

This work was supported by the DFG priority programme 1104 'Colloidal Magnetic Fluids' and the Max Planck Institute for Marine Microbiology, Bremen. We thank numerous colleagues for most valuable collaborations. The contribution of former graduate students to the presented work is gratefully acknowledged. 


\section{References}

[1] Bazylinski D A and Frankel R B 2004 Nat. Rev. Microbiol. 2217

[2] Flies C, Peplies J and Schüler D 2005 Appl. Environ. Microbiol. 712723

[3] Schüler D and Frankel R B 1999 Appl. Microbiol. Biotechnol. 52464

[4] Blakemore R P and Frankel R B 1981 Sci. Am. 24542

[5] Komeili A, Li Z, Newman D K and Jensen G J 2006 Science 311242

[6] Scheffel A, Gruska M, Faivre D, Linaroudis A, Graumann P L, Plitzko J M and Schüler D 2006 Nature 440110

[7] Moskowitz B M 1995 Rev. Geophys. 33123

[8] Dunin-Borkowski R E, McCartney M R, Posfai M, Frankel R B, Bazylinski D A and Buseck P R 2001 Eur. J. Mineral. 13671

[9] Schüler D 1999 J. Mol. Microbiol. Biotechnol. 179

[10] Devouard B, Posfai M, Hua X, Bazylinski D A, Frankel R B and Buseck P R 1998 Am. Mineral. 831387

[11] Schübbe S, Kube M, Scheffel A, Wawer C, Heyen U, Meyerdierks A, Madkour M, Mayer F, Reinhardt R and Schüler D 2003 J. Bacteriol. 1855779

[12] Ullrich S, Kube M, Schübbe S, Reinhardt R and Schüler D 2005 J. Bacteriol. 1877176

[13] Cornell R M and Schwertmann U 2003 The Iron Oxides-Structure, Properties, Reactions, Occurrences and Uses 2nd edn (Weinheim: Wiley-VCH)

[14] Gorby Y A, Beveridge T J and Blakemore R P 1988 J. Bacteriol. 170834

[15] Schüler D 2002 Int. Microbiol. 5209

[16] Yoza B, Arakaki A and Matsunaga T 2003 J. Biotechnol. 101219

[17] Yoza B, Arakaki A, Maruyama K, Takeyama H and Matsunaga T 2003 J. Biosci. Bioeng. 9521

[18] Sode K, Kudo S, Sakaguchi T, Nakamura N and Matsunaga T 1993 Biotechnol. Tech. 7688

[19] Matsunaga T, Nakayama H, Okochi M and Takeyama H 2001 Biotechnol. Bioeng. 73400

[20] Matsunaga T and Kamiya S 1987 Appl. Microbiol. Biotechnol. 26328

[21] Nakamura N and Matsunaga T 1993 Anal. Chim. Acta 281585

[22] Tanaka T and Matsunaga T 2000 Anal. Chem. 723518

[23] Yoshino T, Takahashi M, Takeyama H, Okamura Y, Kato F and Matsunaga T 2004 Appl. Environ. Microbiol. $\mathbf{7 0} 2880$

[24] Tanaka T, Takeda H, Ueki F, Obata K, Tajima H, Takeyama H, Goda Y, Fujimoto S and Matsunaga T 2004 J. Biotechnol. 108153

[25] Kuhara M, Takeyama H, Tanaka T and Matsunaga T 2004 Anal. Chem. 766207

[26] Tanaka T, Maruyama K, Yoda K, Nemoto E, Udagawa Y, Nakayama H, Takeyama H and Matsunaga T 2003 Biosensors Bioelectron. 19325

[27] Amemiya Y, Tanaka T, Yoza B and Matsunaga T 2005 J. Biotechnol. 120308

[28] Banerjee I A, Yu L, Shima M, Yoshino T, Takeyama H, Matsunaga T and Matsui H 2005 Adv. Mater. 171128

[29] Schultheiss D, Kube M and Schüler D 2004 Appl. Environ. Microbiol. 703624

[30] Heyen U and Schüler D 2003 Appl. Microbiol. Biotechnol. 61536

[31] Schüler D and Baeuerlein E 1997 J. Physique IV 7647

[32] Grünberg K, Müller E C, Otto A, Reszka R, Linder D, Kube M, Reinhardt R and Schüler D 2004 Appl. Eviron. Microbiol. 701040

[33] Grünberg K, Wawer C, Tebo B M and Schüler D 2001 Appl. Environ. Microbiol. 674573

[34] Laemmli U K 1970 Nature 227680

[35] Schägger H and von Jagow G 1987 Anal. Biochem. 166368

[36] Nies D H 2003 FEMS Microbiol. Rev. 27313

[37] Clausen T, Southan C and Ehrmann M 2002 Mol. Cell 10443

[38] Schüler D 2004 Arch. Microbiol. 1811

[39] Arakaki A, Webb J and Matsunaga T 2003 J. Biol. Chem. 2788745

[40] Dunin-Borkowski R E, McCartney M R, Frankel R B, Bazylinski D A, Posfai M and Buseck P R 1998 Science 2821868

[41] Hergt R, Hiergeist R, Zeisberger M, Schüler D, Heyen U, Hilger I and Kaiser W A 2005 J. Magn. Magn. Mater. 29380

[42] Eberbeck D, Janke V, Hartwig S, Heyen U, Schüler D, Albrecht M and Trahms L 2005 J. Magn. Magn. Mater. 28970

[43] Albrecht M, Janke V, Sievers S, Siegner U, Schüler D and Heyen U 2005 J. Magn. Magn. Mater. 290/291 269

[44] Hoell A, Wiedenmann A, Heyen U and Schüler D 2004 Physica B 350309

[45] Herborn C U, Papanikolaou N, Reszka R, Grünberg K, Schüler D and Debatin J F 2003 Rofo Fortschr. Geb. Rontgenstr. Neuen Bildgeb. Verfahr. 175830 
[46] Hartung A, Trost R, Lisy R, Hilger I, Kaiser W A and Reichenbach J R 2006 Scientific and Clinical Application of Magnetic Carriers (Krems, Austria 2006)

[47] Hilger I, Hergt R and Kaiser W A 2000 Invest. Radiol. 35170

[48] Komeili A, Vali H, Beveridge T J and Newman D K 2004 Proc. Natl Acad. Sci. USA 1013839

[49] Schultheiss D, Handrick R, Jendrossek D, Hanzlik M and Schüler D 2005 J. Bacteriol. 1872416

[50] Lang C and Schüler D 2006 Microbial Bionanotechnology: Biological Self-Assembly Systems and BiopolymerBased Nanostructures ed B Rehm (Wymondham: Horizon Scientific Press) p 107

[51] Schultheiss D and Schüler D 2003 Arch. Microbiol. 17989

[52] Junge K et al unpublished 